\title{
Castration failure in prostate carcinoma due to a functioning adrenocortical carcinoma
}

\author{
Vishal Navani@1, James F Lynam ${ }^{1,2}$, Steven Smith³, Christine J O'Neill2,4,5 and Christopher W Rowe ${ }^{2,6}$ \\ 1'Department of Medical Oncology, Calvary Mater Hospital, Newcastle, New South Wales, Australia, 2School of \\ Medicine and Public Health, University of Newcastle, Newcastle, New South Wales, Australia, ${ }^{3}$ Department of Nuclear \\ Medicine, John Hunter Hospital, Newcastle, New South Wales, Australia, 4 Surgical Services, John Hunter Hospital, \\ Newcastle, New South Wales, Australia, 5 University of Newcastle, Newcastle, New South Wales, Australia, and \\ ${ }^{6}$ Department of Endocrinology, John Hunter Hospital, Newcastle, New South Wales, Australia \\ Correspondence \\ should be addressed \\ to C W Rowe \\ Email \\ christopher.rowe@ \\ hnehealth.nsw.gov.au
}

\section{Summary}

We report concurrent metastatic prostatic adenocarcinoma (PC) and functioning androgen-secreting adrenocortical carcinoma (ACC) in a 77-year-old man. The failure to achieve adequate biochemical castration via androgen deprivation therapy (ADT) as treatment for PC metastases, together with elevated DHEA-S, androstenedione, and discordant adrenal tracer uptake on FDG-PET and PSMA-PET, suggested the presence of a concurrent functional primary adrenal malignancy. On histopathological analysis, scant foci of PC were present throughout the ACC specimen. Castration was achieved post adrenalectomy with concurrent drop in prostate-specific antigen. We outline the literature regarding failure of testosterone suppression on ADT and salient points regarding diagnostic workup of functioning adrenal malignancies.

\section{Learning points:}

- Failure to achieve castration with androgen deprivation therapy is rare and should prompt careful review to identify the underlying cause.

- All adrenal lesions should be evaluated for hormone production, as well as assessed for risk of malignancy (either primary or secondary).

- Adrenocortical carcinomas are commonly functional, and can secrete steroid hormones or their precursors (androgens, progestogens, glucocorticoids and mineralocorticoids).

- In this case, a co-incident, androgen-producing adrenocortical carcinoma was the cause of failure of testosterone suppression from androgen deprivation therapy as treatment for metastatic prostate cancer. Pathological adrenal androgen production contributed to the progression of prostate cancer.

\section{Background}

The primacy of androgen signalling in the development of prostate cancer is well defined (1). Androgen deprivation therapy (ADT) has been the cornerstone of metastatic prostatic adenocarcinoma (mPC) management for decades, with prolonged survival achieved via pharmacological therapies to lower circulating testosterone (2). The majority of advanced prostate cancer patients respond initially to ADT, with a target castrate level defined as testosterone $<0.7 \mathrm{nmol} / \mathrm{L}$. Failure to achieve a castrate level of testosterone is rare, with only isolated case reports in the literature (3). Here we present the first reported case of failure of testosterone suppression despite medical ADT in a patient with $\mathrm{mPC}$ due to a concurrent androgen-secreting adrenocortical carcinoma. 


\section{Case presentation}

A 77-year-old man presented with a 12-month history of worsening mid-thoracic back pain. Initial workup revealed elevation of serum prostate-specific antigen (PSA) at 490 $\mu \mathrm{g} / \mathrm{L}$ (reference range $(\mathrm{RR}):<7.5)$. A transrectal prostate biopsy confirmed Gleason $5+4=9$ prostatic adenocarcinoma (PC). Staging contrast-enhanced computed tomography (CT) showed an enlarged prostate, widespread sclerotic bony lesions, malignant retroperitoneal lymphadenopathy and visceral lung lesions. Additionally, the left adrenal gland was enlarged by a heterogenous, 68 x $45 \times 45 \mathrm{~mm}$ mass without calcification but with elevated pre-contrast density (20-30 Hounsfield units), with significant contrast uptake and slow washout (Fig. 1E and F). A clinical diagnosis of metastatic prostate cancer was made, with the identified lesions ascribed to prostate cancer metastases, and he was commenced on ADT with leuprorelin (GnRH analogue) and cyproterone acetate (androgen receptor antagonist).

Following 8 weeks of ADT therapy, PSA had fallen to $120 \mu \mathrm{g} / \mathrm{L}$; however, serum testosterone remained elevated at $21.5 \mathrm{nmol} / \mathrm{L}$ (reference range 8-30), despite undetectable
A

B
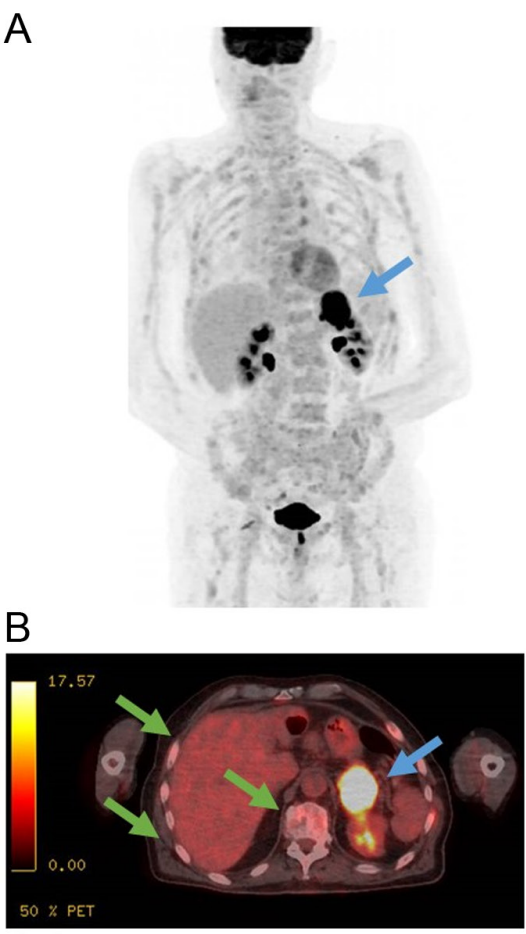

E

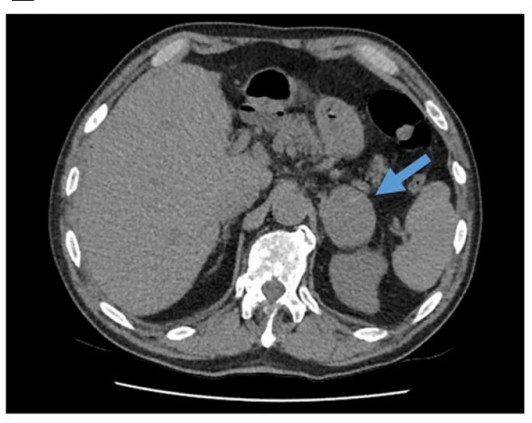

C
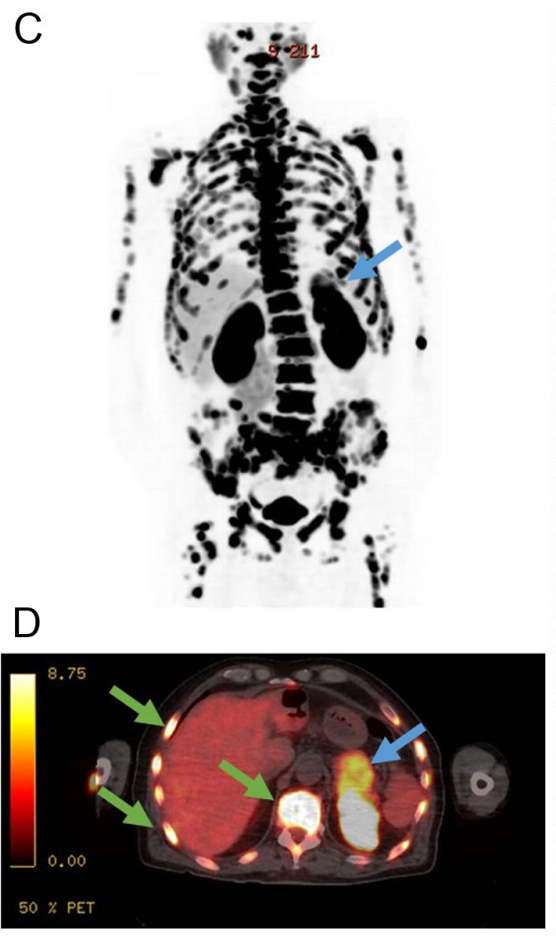

$\mathrm{F}$

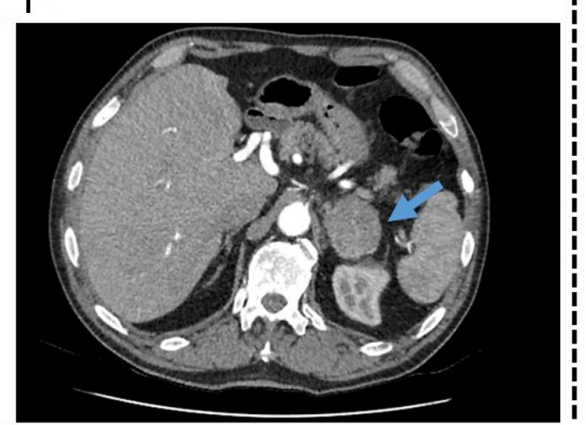

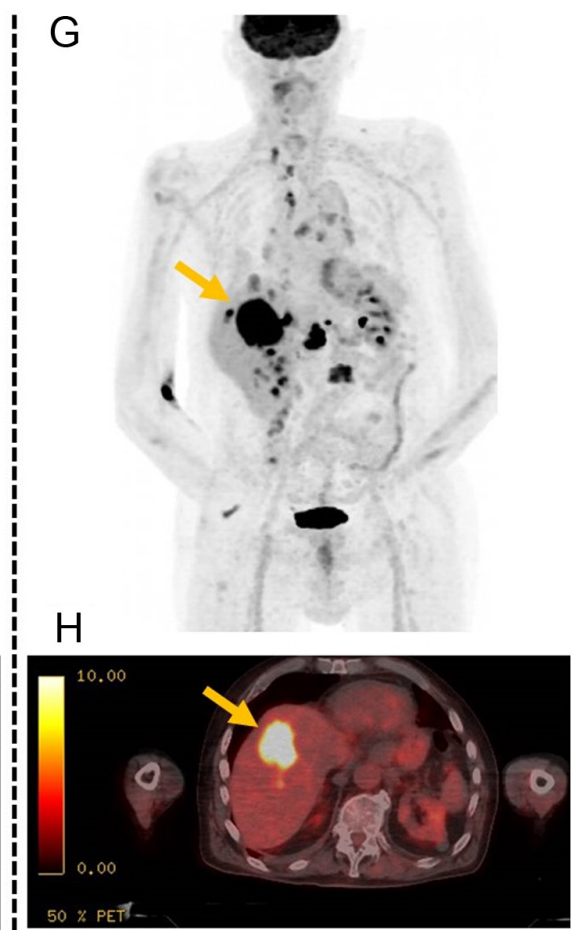

\section{Figure 1}

(A and B) Pre-operative 18F-FDG-PET, cine (A) and axial-fused (B), demonstrating focal intense uptake in left adrenal gland (blue arrow), with mimimal uptake in the skeleton body (green arrows). (C and D) Pre-operative 68Ga-PSMA-PET, cine (C) and axial-fused (D), demonstrating modest uptake in left adrenal gland (blue arrow) but intense widespread uptake in the skeleton and vertebral body (green arrows), consistent with metastatic prostatic carcinoma. (E and F) Pre-operative axial computed tomography without intravenous contrast (E) and with contrast in the arterial phase (F), demonstrating a large, irregular, heterogeneous left adrenal gland with pre-contrast density 20-30 Hounsefield units. The right adrenal gland can be seen as normal. (G and H) 12 months post-operative 18F-FDG-PET, cine (G) and axial-fused (H) showing a new, intensely hypermetabolic liver metastases (gold arrow). Thoracic and abdominal nodal metastases are also seen (G). 
levels of follicular stimulating hormone (FSH) and luteinising hormone (LH) (Table 1). Given persistently elevated testosterone, the GnRH analogue was switched to triptorelin. Despite this, testosterone remained persistently elevated at $23 \mathrm{nmol} / \mathrm{L}$, with PSA $98 \mu \mathrm{g} / \mathrm{L}$. Repeat staging CT showed growth in the left adrenal mass (now 78 x $50 \mathrm{~mm}$ ) with local extension towards the posterior surface of the stomach. Other sites of metastatic disease were stable.

\section{Investigation}

The differential diagnosis for the enlarging left adrenal mass was reviewed. Clinical examination revealed no evidence of androgen or cortisol excess. He appeared eugonadal, uncommon on ADT. An adrenal hormonal panel showed, in addition to persistently elevated testosterone performed in two separate assays, elevated DHEA-S at $13 \mu \mathrm{mol} / \mathrm{L}$ (RR: <10) and androstenedione $28 \mathrm{nmol} / \mathrm{L}$ (RR: 2.1-11). Evidence for autonomous cortisol excretion was equivocal with normal $24 \mathrm{~h}$ urinary free cortisol (159 nmol/24 h, RR: <270) but failure of suppression of cortisol following $1 \mathrm{mg}$ overnight dexamethasone suppression test (basal cortisol $437 \mathrm{nmol} / \mathrm{L}$ with ACTH $0.6 \mathrm{nmol} / \mathrm{L}$ (RR: 1-10); postsuppression cortisol $363 \mathrm{nmol} / \mathrm{L}$, normal: <50 nmol/L). Plasma metanephrines and normetanephrines were negative. The presumptive diagnosis for the left adrenal lesion was revised to androgen-secreting adrenocortical carcinoma (ACC).

Functional imaging with fluorine-18 fluorodeoxyglucose (FDG) positron emission tomography (PET) scan identified intense radiopharmaceutical uptake within the left adrenal and low-grade radiopharmaceutical uptake at sites of skeletal metastases (Fig. 1A and B). By contrast, a Gallium- 68 prostate-specific membrane antigen (PSMA) PET showed moderate radiopharmaceutical uptake within the ACC and avid radiopharmaceutical uptake within the metastases throughout the skeleton (Fig. 1C and D). This, combined with the structural stability of the skeletal lesions, was considered supportive that the metastatic disease was prostatic carcinoma and that the presumed ACC remained localised to the adrenal bed.

\section{Treatment}

In view of the high lethality of untreated ACC, as well as the strong clinical suspicion that androgen secretion from the adrenal tumour was driving progression of metastatic prostatic cancer, he proceeded to left adrenalectomy. Given the presence of prostatic cancer metastases, a laparoscopic approach was attempted to minimise morbidity. However, the tumour was highly vascular and difficult to mobilise, requiring conversion to an open procedure. The adrenal tumour was removed intact with marginal clearance but preservation of all surrounding structures. There were no perioperative complications.

Histological examination showed a pT3 high-grade adrenocortical carcinoma (Ki-67: 40\%), 80 mm in maximal length, invading perivesical fat but with clear resection margins. Scant foci of metastatic PC were observed within the ACC parenchyma (Fig. 2). These concomitant carcinomas were confirmed on immunohistochemical staining, with mutually exclusive synaptophysin (Fig. 2C) and inhibin expression in ACC (Fig. 2C) and prostate-specific antigen expression in mPC (Fig. 2D).

Table 1 Hormonal profile prior to and following treatment for adrenocortical carcinoma.

\begin{tabular}{|c|c|c|c|c|c|c|c|c|}
\hline & \multicolumn{2}{|c|}{ Pre-adrenalectomy, day } & \multicolumn{5}{|c|}{ Post-adrenalectomy, day } & \multirow{2}{*}{$\begin{array}{l}\text { Reference } \\
\text { range }\end{array}$} \\
\hline & D-120 & D-90 & $\mathrm{D}+30$ & $D+180$ & $D+310$ & $\mathrm{D}+400$ & $D+430$ & \\
\hline Event & $\begin{array}{l}\text { Prostate CA } \\
\text { diagnosed }\end{array}$ & On ADT & ADT + Mitotane & ADT + Mitotane & ADT + Mitotane & $\begin{array}{c}\text { Mitotane } \\
\text { ceased }\end{array}$ & $\begin{array}{l}\text { Palliative } \\
\text { pathway }\end{array}$ & \\
\hline PSA, $\mu g / L$ & 490 & $98-120$ & 16.0 & 0.20 & 0.92 & 5.4 & 4.7 & $0.3-7.5$ \\
\hline Testosterone, $\mu \mathrm{mol} / \mathrm{L}$ & & $21-23$ & $<0.4$ & $<0.4$ & $<0.4$ & 7.3 & 16.9 & $8-30$ \\
\hline DHEA-S, $\mu \mathrm{mol} / \mathrm{L}$ & & $10-13$ & 0.9 & 0.1 & 0.6 & 15.8 & 25.4 & $<10$ \\
\hline $\begin{array}{l}\text { Androstenedione, } \\
\mathrm{nmol} / \mathrm{L}\end{array}$ & & 28 & 0.9 & 0.9 & NA & 32.1 & 55.0 & $2-11$ \\
\hline ACTH, pmol/L & $<0.1$ & & & & & & & $1.0-10.8$ \\
\hline \multicolumn{9}{|l|}{ Cortisol, nmol/L } \\
\hline 8 am, basal & 437 & & & 91 & & & & \\
\hline post $1 \mathrm{mg}$ DST & 363 & & & & & & & \\
\hline
\end{tabular}

Day (D) is with reference to day of adrenalectomy Day=0; ADT, androgen deprivation therapy; DST, cortisol level following 1 mg overnight dexamethasone suppression test; PSA, prostate-specific antigen. 

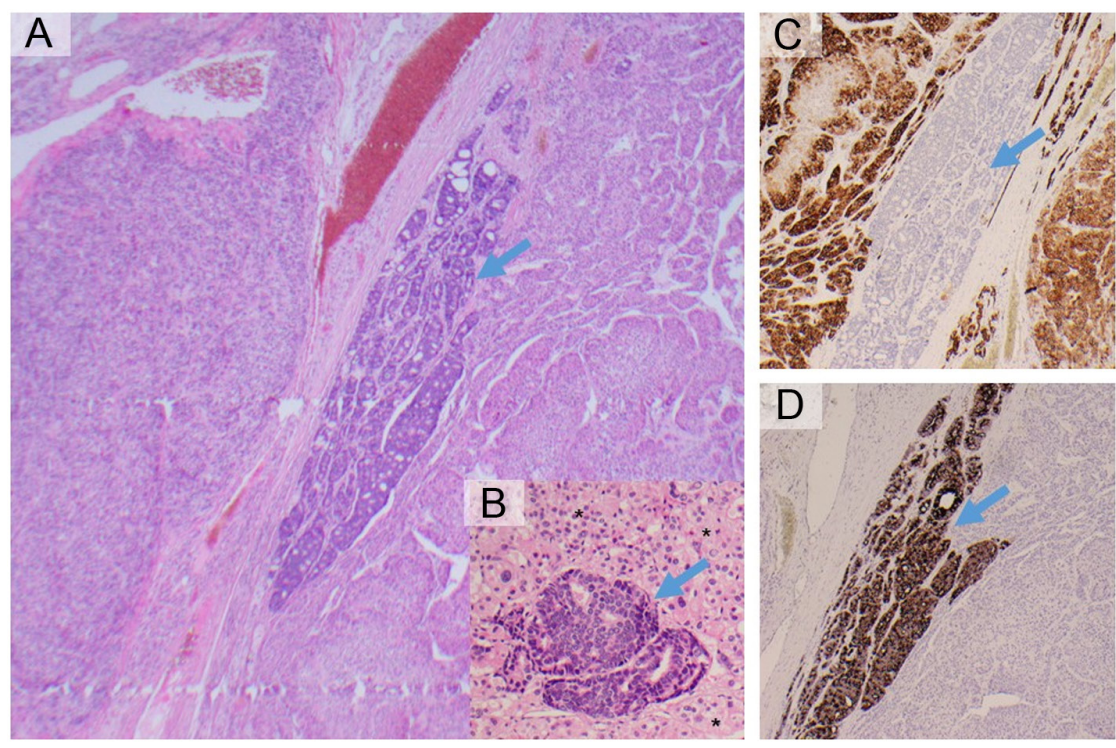

\section{Figure 2}

Histopathology of adrenal gland. (A) Low- power haematoxylin \& eosin stain, showing a small focus of prostatic adenocarcinoma (arrow) surrounded by adrenocortical carcinoma. (B) High- power haematoxylin \& eosin stain, showing typical nests of large adrenocortical cells with marked nuclear pleomorphism and abundant pale eosinophilic cytoplasm (asterisks). In the centre (arrow) a focus of metastatic prostatic carcinoma, demonstrating cribriform structures and prominent nucleoili. (C) Low-power section of block in (A),

immunohistochemically stained for synaptophysin. Intense staining of the adrenocortical carcinoma is demonstrated (brown DAB stain), with negative stain of the prostate adenocarcinoma metastases (arrow). (D) Low-power section of block in (A), immunohistochemically stained for prostatespecific antigen. Intense staining of the prostatic adenocarcinoma is demonstrated (brown DAB stain, arrow), with negative stain of the surrounding adrenocortical carcincoma.

\section{Outcome and follow-up}

Three weeks post-operatively PSA fell to $30 \mu \mathrm{g} / \mathrm{L}$, with a now appropriately supressed testosterone at $<0.4 \mathrm{nmol} / \mathrm{L}$ (Table 1). Six weeks post-operative PSA was $16 \mu \mathrm{g} / \mathrm{L}$ with low DHEA-S $0.9 \mu \mathrm{mol} / \mathrm{L}$, androstenedione $0.9 \mathrm{nmol} / \mathrm{L}$ and testosterone $<0.4 \mathrm{nmol} / \mathrm{L}$. He received adjuvant irradiation to his adrenal bed. Given the limited prognosis even in resected stage III ACC, with a 24\% 5-year survival only, the consensus multidisciplinary team decision was to prioritise adjuvant treatment for his ACC over intensifying systemic therapy for his $\mathrm{MPC}$, and adjuvant mitotane was commenced. Standard of care GnRH-based ADT for $\mathrm{mPC}$, now in the metastatic hormone-sensitive state (mHSPC), was continued. The patient remained stable on mitotane and ADT, in clinical and biochemical remission from ACC, for 12 months after adrenalectomy. A surveillance 18F-FDG PET at 6 months showed no evidence of recurrent disease. However, 18F-FDGPET performed 13 months after adrenalectomy, and coincident with the development of thoracic back pain and deconditioning, demonstrated multiple FDG-avid foci consistent with metastatic ACC (Fig. 1G and H). Concurrently, levels of adrenal androgens began to increase, consistent with functional ACC metastases (Table 1), with corresponding increases in PSA, consistent with stimulation of mPC by adrenal androgens. Due to a declining functional status, palliative chemotherapy was not initiated. Mitotane therapy was ceased, and palliative care was enhanced. He died 16 months after adrenalectomy and 20 months after diagnosis of metastatic prostate cancer.

\section{Discussion}

This case represents an unusual cause of failure of medical ADT due to a functioning, androgen-secreting, ACC. ADT, via medical or surgical approaches, has been a cornerstone of treatment for advanced prostate cancer since the first work outlining the sensitivity of this disease to androgens. In this case, adrenal androgens contributed to the progression of $\mathrm{mPC}$, given the correlation between PSA and androgen status both at initial diagnosis and at ACC recurrence.

Given gonadotrophin receptor agonists/antagonists are widely effective medical approaches to achieving castration, they are established first-line therapy for androgen deprivation in advanced prostate cancer. There are isolated case reports of primary failure of suppression of testosterone secretion in this setting (4), with various possible explanations offered, ranging from anti-drug antibodies, incorrect administration or rapid metabolism of drug. Persistent elevation of LH suggests incorrect administration. A single prospective observational study reported that the rate of failure of ADT to suppress testosterone was $5 \%(n=38)$; associated with obesity, and resolved following orchidectomy (5). A single case of a functional gonadotrophin-secreting pituitary adenoma has been reported (6).

Adrenocortical carcinomas (ACC) are rare, with a bimodal age distribution affecting young children and those in middle age, with a slight female preponderance. Sixty per cent are sufficiently secretory to present with symptoms of hormone excess, such as Cushing's 
syndrome, virilisation or hyperaldosteronism. Nonfunctioning tumours are more likely to present with symptoms of local tumour growth such as flank pain, with $10 \%$ found incidentally. Most adrenal incidentalomas are due to benign non-functioning adrenocortical adenomas, but must be distinguished from functional adenomas, metastases, phaeochromocytoma, adjacent non-adrenal pathology and the much rarer adrenocortical carcinoma. Benign lipid-rich adenomas have a typical CT appearance with low attenuation (less than 10 Hounsfield units), rapid washout of contrast ( $>50 \%$ at $10 \mathrm{~min}$ ), smooth margins and a homogenous appearance. ACC are generally larger (most $>4 \mathrm{~cm}$ at diagnosis), with higher attenuation, heterogenous appearance with calcification and occasional evidence of invasion. Specificity for malignancy rises with increasing size. Distinguishing primary adrenal carcinoma from metastases can be challenging; however, the presence of hormonal hypersecretion (glucocorticoids, mineralocorticoids or androgens) is an important indicator of a primary adrenal tumour.

Given the similarity of preferential sites of metastatic disease for PC and adrenocortical carcinoma, namely bone, lymph nodes, lung and liver, functional imaging studies are helpful to differentiate likely aetiologies. Adrenal metastases from PC are rare, with a real-world observational cohort of over 74000 patients, incidence to be only $1 \%$ after 12 years of follow-up (7).

FDG PET is a well-established modality to distinguish benign and malignant adrenal lesions. For example, in a large study with 1391 adrenal lesions, FDG PET was able to differentiate malignant from benign lesions with a sensitivity of $97 \%$ and a specificity of $91 \%$ (8).

It is now recognised that PSMA is poorly named, as it is now understood to be a glutamate carboxypeptidase receptor II that is overexpressed in a wide variety of malignancies. Crowley and colleagues previously have raised the potential of using PSMA to both diagnose and differentiate ACC from other benign and malignant adrenal tumours by a small sample that shows ACC expressed PSMA in 78\% of the ACC neovasculature, where it was absent in normal adrenal glands and only present in $3 \%$ of adenoma neovasculature (8).

In this case, a combination of the FDG PET and PSMA PET was able to characterise two different patterns of malignant disease; the ACC which was intensely FDG avid and moderately PSMA avid and the metastatic prostate cancer which was mildly FDG avid and intensely PSMA avid. The combination of scans was able to support the case that the ACC was not metastatic and suggested that surgical resection of the ACC may not only improve outcome for the prostate cancer (by reducing androgen secretion) but also may improve outcome from the primary adrenal malignancy by resecting all known disease.

Crowley and colleagues also postulated the potential of using lutetium and actinium PSMA labelled radioisotopes as a treatment option for ACC (9). In this case where there is a synchronous prostate cancer, PSMA radionuclide therapy would have the advantage of potentially working for both the distant metastatic prostate cancer and the future recurrent or metastatic ACC.

Mitotane, an insecticide isomer, is an adrenolytic drug used to treat ACC. It has a cytotoxic effect on mitochondria in the adrenocortical cells of the reticularis and fasciculate. Mitotane inhibits activity in the cholesterol side chain cleavage (CYP11A1) enzyme and CYP11B activity within ACC, affecting adrenal cortisol and testosterone biosynthesis. Indeed, when used in the adjuvant setting for ACC, mitotane has been found to decrease DHEA-S concurrent with cortisol, whilst being associated with a clinical syndrome of hypogonadism. Extra-adrenal actions of mitotane and increased sex hormone-binding globulin (SHBG) and resultant decreased free testosterone, together with inhibition of $5 \alpha$-reductase, contribute to known hypogonadic sequelae. A human hepatoma cell line model utilising male patients on adjuvant mitotane for ACC suggested reduced total and free [testosterone], concurrent with oestrogen- dependent increase in SHBG, alongside with low LH suggesting pituitary activity of mitotane. This hypogonadic state induced by the pluripotent activity of mitotane may provide a beneficial impact on the clinical course of our patient's castrate-sensitive PC, independently of medical ADT and resection of his androgen-producing ACC. Murine mHRPC xenograft studies have shown that adrenally derived androgens are key drivers of tumour growth, with surgical bilateral adrenalectomy reducing tumour volumes (10).

This unusual presentation of a concurrent functioning ACC and mPC illustrates the complex endocrine interplay of both diseases. The failure of castration via ADT is a rare occurrence and should prompt careful evaluation of a wide range of differential diagnoses.

\section{Declaration of interest}

The authors declare that there is no conflict of interest that could be perceived as prejudicing the impartiality of the research reported.

\section{Funding}

This research did not receive any specific grant from any funding agency in the public, commercial or not-for-profit sector. 


\section{Patient consent}

Written informed consent for publication of their clinical details and/or clinical images was obtained from the patient.

\section{Author contribution statement}

All authors were involved in the care of the patient and were involved in the preparation of the manuscript and have approved the final form for publication.

\section{Acknowledgements}

The authors would like to thank Dr Gagandeep Sandhu (Department of Anatomical Pathology, NSW Health Pathology Hunter) for providing histopathological photographs.

\section{References}

1 Ruizeveld de Winter JA, Janssen PJ, Sleddens HM, VerleunMooijman MC, Trapman J, Brinkmann AO, Santerse AB, Schröder FH $\&$ van der Kwast TH. Androgen receptor status in localized and locally progressive hormone refractory human prostate cancer. American Journal of Pathology 1994144 735-746.

2 Perlmutter MA \& Lepor H. Androgen deprivation therapy in the treatment of advanced prostate cancer. Reviews in Urology 20079 (Supplement 1) S3-S8.

3 Koh Y, Kawashima A, Ujike T, Nagahara A, Fujita K, Kiuchi H, Imamura R, Miyagawa Y, Nonomura N \& Uemura M. Failure to achieve castrate level of serum testosterone during luteinizing hormonereleasing hormone agonist therapy in a patient with prostate cancer.
Anti-Cancer Drugs 202031 1099-1102. (https://doi.org/10.1097/ CAD.0000000000000986)

4 Jorion JL. Idiopathic resistance to luteinizing hormone-releasing hormone analogue in a patient with prostatic carcinoma. Journal of Urology 1992148 1539-1540. (https://doi.org/10.1016/s00225347(17)36962-8)

5 Oefelein MG \& Cornum R. Failure to achieve castrate levels of testosterone during luteinizing hormone releasing hormone agonist therapy: the case for monitoring serum testosterone and a treatment decision algorithm. Journal of Urology 2000164 726-729. (https://doi org/10.1097/00005392-200009010-00025)

6 Ogan K, Berger M \& Ball R. Gonadotropin releasing hormone analogue antiandrogen failure secondary to a pituitary adenoma. Journal of Urology 1998160 497-498. (https://doi.org/10.1016/S00225347(01)62937-9)

7 Gandaglia G, Abdollah F, Schiffmann J, Trudeau V, Shariat SF, Kim SP, Perrotte P, Montorsi F, Briganti A, Trinh QD, et al. Distribution of metastatic sites in patients with prostate cancer: a population-based analysis. Prostate 201474 210-216. (https://doi.org/10.1002/pros.22742)

8 Boland GW, Dwamena BA, Jagtiani Sangwaiya M, Goehler AG, Blake MA, Hahn PF, Scott JA \& Kalra MK. Characterization of adrenal masses by using FDG PET: a systematic review and meta-analysis of diagnostic test performance. Radiology 2011259 117-126. (https://doi. org/10.1148/radiol.11100569)

9 Crowley MJ, Scognamiglio T, Liu YF, Kleiman DA, Beninato T, Aronova A, Liu H, Jhanwar YS, Molina A, Tagawa ST, et al. Prostatespecific membrane antigen is a potential antiangiogenic target in adrenocortical carcinoma. Journal of Clinical Endocrinology and Metabolism 2016101 981-987. (https://doi.org/10.1210/jc.2015-4021)

10 Mostaghel EA, Zhang A, Hernandez S, Marck BT, Zhang X, Tamae D, Biehl HE, Tretiakova M, Bartlett J, Burns J, et al. Contribution of adrenal glands to intratumor androgens and growth of castrationresistant prostate cancer. Clinical Cancer Research 201925 426-439. (https://doi.org/10.1158/1078-0432.CCR-18-1431)

Received in final form 11 August 2021

Accepted 28 September 2021 